\title{
A Classification \\ of Special Riemannian 3-Manifolds with Distinct Constant Ricci Eigenvalues
}

\author{
O. Kowalski and F. Prüfer
}

\begin{abstract}
All Riemannian 3-manifolds with distinct constant Ricci eigenvalues and satisfying some additional geometrical conditions are classified in an explicit form. One obtains locally homogeneous spaces and two different classes of locally non-homogeneous spaces in this way.
\end{abstract}

Keywords: Curvature homogeneous 3-manifolds, constant Ricci eigenvalues

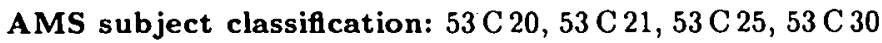

\section{Introduction}

According to I. M. Singer [14] a Riemannian manifold $(M, g)$ is said to be curvature homogeneous if, for every two points $p, q \in M$, there is a linear isometry $F: T_{p} M \rightarrow$ $T_{q} M$ between the corresponding tangent spaces such that $F^{*} R_{q}=R_{p}$ (where $R$ denotes the curvature tensor of type $(0,4)$ ). Note that a (locally) homogeneous Riemannian manifold is automatically curvature homogeneous. Explicit locally non-homogeneous examples have been constructed by many authors (see [4 - 6], [8 - 10], [12], [16] and [17]; see especially [9] and [10] for more complete references).

For 3-dimensional Riemannian manifolds $(M, g)$ the following simple criterion holds:

A Riemannian manifold $(M, g)$ is curvature homogeneous if and only if all Ricci eigenvalues of $(M, g)$ are constant.

Thus the problem to classify all 3-dimensional Riemannian manifolds with prescribed constant Ricci eigenvalues is of considerable interest. This problem was investigated already in 1916 by L. Bianchi [1] who made a classification under a strong additional hypothesis of "normality". He found only some homogeneous Riemannian spaces as solutions. On the other hand, the following conclusion follows from an observation by J. Milnor [11] and a result by K. Sekigawa [13]:

For a homogeneous Riemannian S-manifold $(M, g)$, the signature of the Ricci tensor is never equal to $(+,+,-)$ and to $(+, 0,-)$.

O. Kowalski: Charles Univ., Math. Institute, Sokolovská 83, 18600 Praha, Czech Republic. The research was partly supported by the grant GA CR 201/93/0469.

F. Prüfer: Univ. Leipzig, Math. Institut, Augustuspl. 10/11, PF 920, D - 04109 Leipzig

ISSN 0232-2064 / \$2.50 C Heldermann Verlag Berlin 
Let us describe shortly what is known about the problem at the present. Let $\rho_{1}, \rho_{2}, \rho_{3}$ denote the constant Ricci eigenvalues of $(M, g)$. The case in which all Ricci eigenvalues are equal is trivial - we obtain only spaces of constant curvature. The case $\rho_{1}=\rho_{2} \neq \rho_{3}$ was solved completely by the first author in [4 - 5]. The answer is that the local isometry classes of the corresponding metrics always depend on two arbitrary functions of one variable. (Notice that the local isometry classes of locally homogeneous spaces in dimension three depend only on a finite number of parameters.) In the same papers, some quasi-explicit formulas were given for all the solutions.

The most difficult and most interesting case is that of distinct constant Ricci eigenvalues. In 1991, K. Yamato [17] presented a broad class of explicit examples - these are complete Riemannian metrics given on $\mathbb{R}^{3}$. Such metrics exist only if the Ricci eigenvalues satisfy certain system of inequalities. In [6] the author gave an easy modification of the Yamato examples. The new metrics are not necessarily complete but the range of the possible Ricci eigenvalues is considerably larger (this includes all Ricci tensors with the signatures $(+,+,-)$ and $(+, 0,-)$, but still not all of the possible cases).

Recently, A. Spiro and F. Tricerri [15] proved the following results:

a) For each triplet of prescribed distinct constant Ricci eigenvalues, there exists at least one corresponding Riemannian metric (1992).

b) The local isometry classes of all such metrics depend on an infinite number of parameters (1993).

(In 1981, DeTurck [2] proved the local existence of a Riemannian metric with the prescribed non-singular Ricci tensor $R_{i j}$. Let us mention that his method cannot be directly applied to the problem of prescribed Ricci eigenvalues.)

In a previous paper [7], the present authors gave explicit examples of locally nonhomogeneous metrics on $\mathbb{R}^{3}$ with any prescribed distinct constant Ricci eigenvalues. These examples can be considered as "generalized Yamato examples".

In this article we use the same method for solving a special classification problem. In this classification we obtain locally homogeneous spaces, all generalized Yamato examples and also a new class of locally non-homogeneous examples.

\section{A classification theorem}

In the following we assume that $(M, g)$ is a Riemannian manifold of class $C^{\infty}$ and $\nabla$ will denote the Levi-Civita connection of $(M, g)$. Suppose that all Ricci eigenvalues (i.e. principal Ricci curvatures) $\rho_{1}, \rho_{2}, \rho_{3}$ are distinct and constant. Locally, one can always find a smooth orthonormal frame $\left\{E_{1}, E_{2}, E_{3}\right\}$ consisting of the eigenvectors of the Ricci tensor $\rho$, respectively. We get with respect to this moving frame

$$
\rho_{i i}=\rho_{i} \quad(i=1,2,3) \quad \text { and } \quad \rho_{i j}=0 \quad(i \neq j) .
$$

For the corresponding components of the Riemannian curvature tensor $R$ we have

$$
\begin{aligned}
& R_{1212}=\lambda_{3}, R_{1313}=\lambda_{2}, R_{2323}=\lambda_{1} \quad\left(\lambda_{1}, \lambda_{2}, \lambda_{3}-\text { constants }\right) \\
& \ddot{R}_{i j k l}=0 \text { if at least three indices are distinct. }
\end{aligned}
$$


Moreover

$$
\lambda_{i}-\lambda_{j}=-\left(\rho_{i}-\rho_{j}\right) \quad(i, j=1,2,3)
$$

because

$$
\rho_{1}=\lambda_{2}+\lambda_{3}, \quad \rho_{2}=\lambda_{1}+\lambda_{3}, \quad \rho_{3}=\lambda_{1}+\lambda_{2} .
$$

Furthermore we put

$$
\alpha=\frac{\rho_{1}-\rho_{3}}{\rho_{3}-\rho_{2}} .
$$

We see at once that $\alpha \neq 0$ and $\alpha \neq-1$. We shall limit ourselves to a sufficiently small connected neighbourhood $U_{p}$ of a fixed point $p \in M$.

In [7] an adapted system of local coordinates $(w, x, y)$ was introduced on $U_{p}$ such that $E_{3}=\frac{\partial}{\partial y}$. Denoting the connection coefficients by $a_{j k}^{i}=g\left(\nabla_{E_{k}} E_{j}, E_{i}\right)$, we get in such a coordinate system

$$
a_{32}^{2}=\alpha a_{31}^{1}, \quad a_{33}^{2}=(\alpha+1) a_{21}^{1}, \quad a_{33}^{1}=-\left(\frac{\alpha+1}{\alpha}\right) a_{22}^{1} .
$$

We call $(M, g)$ a generalized Yamato space on $U_{p}$ if (with respect to some adapted coordinate system) the $a_{j k}^{i}$ do not depend on $y$ and satisfy the following conditions on $U_{p}$ :

(Y1) $a_{31}^{1}=0, \quad a_{23}^{1}+a_{31}^{2}=0, \quad a_{22}^{1}=0$.

(Y2) $a_{23}^{1}$ is an arbitrary non-constant function of class $C^{\infty}$ on $U_{p}$ such that $a_{23}^{1}(q) \neq 0$ for $q \in U_{p}$.

(Y3) $a_{21}^{1}$ is a smooth function on $U_{p}$ satisfying

(a) $(\alpha+1)\left(a_{21}^{1}\right)^{2}+\left(a_{23}^{1}\right)^{2}=\lambda_{2}$

(b) $d a_{21}^{1}(q) \neq 0$ for all $q \in U_{p}$

(Y4) $a_{32}^{1}$ is a smooth function on $U_{p}$. satisfying $-\alpha a_{23}^{1} a_{32}^{1}=(\alpha+1) \lambda_{3}+\lambda_{2}$.

Conversely, if the $a_{j k}^{i}$ are given by $(Y 1)-(Y 4)$ in our coordinate system, then we can find an explicit Riemannian metric $g$ on $U_{p}$ with the constant Ricci eigenvalues $\rho_{1}, \rho_{2}, \rho_{3}$ and not being locally homogeneous (see [7] for the motivation and the next theorem for more details).

Each generalized Yamato space satisfies, for one of the basic vector fields $E_{\gamma}(\gamma \in$ $\{1,2,3\}$ ), the following two geometrical conditions (in which without loss of generality $\gamma=3$ can be assumed):

(G1) The connection coefficients $a_{j k}^{i}$ are constant along the trajectories of $E_{\gamma}$, i.e. $E_{\gamma}\left(a_{j k}^{i}\right)=0$.

(G2) The local group of local diffeomorphismus defined by $E_{\gamma}$ is volume-preserving, i.e. $\operatorname{div} E_{\gamma}=0$.

In the following, we shall also consider the orthonormal coframe $\left\{\omega^{1}, \omega^{2}, \omega^{3}\right\}$ corresponding to $\left\{E_{1}, E_{2}, E_{3}\right\}$ and call it an adapted orthonormal coframe.

Now we are able to formulate our main result. 
Theorem 1.1. Let $(M, g)$ be a $C^{\infty}$-Riemannian manifold of dimension three with distinct constant Ricci eigenvalues. If $(M, g)$ satisfies the conditions (G1) and (G2) (with $\gamma=3$ ) in a neighbourhood of each point $p \in M$, then there is a dense open subset $U \subset M$ such that, for some neighbourhood $V_{q}$ of any point $q \in U$, one of the following three cases (i) - (iii) occur:

(i) $(M, g)$ restricted to $V_{q}$ is locally isometric to a 9 -dimensional Lie group with a left-invariant metric.

(ii) $(M, g)$ restricted to $V_{q}$ is locally isometric to a generalized Yamato space. This means that the adapted orthonormal coframe $\left\{\omega^{1}, \omega^{2}, \omega^{3}\right\}$ is given with respect to an adapted system $(w, x, y)$ of local coordinates by the formulas

$$
\left.\begin{array}{l}
\omega^{1}=A d w+B d x \\
\omega^{2}=C d w+D d x \\
\omega^{3}=G d w+H d x+d y
\end{array}\right\}
$$

and

$$
\begin{array}{ll}
A=C\left(\varphi_{3}-\varphi_{1}\right) y+A_{0} & B=D\left(\varphi_{3}-\varphi_{1}\right) y+B_{0} \\
C=\frac{\left(\varphi_{1}\right)_{w}^{\prime}}{\left(\alpha \varphi_{1}+(\alpha+2) \varphi_{3}\right) \varphi_{2}} & D=\frac{\left(\varphi_{1}\right)_{x}^{\prime}}{\left(\alpha \varphi_{1}+(\alpha+2) \varphi_{3}\right) \varphi_{2}} \\
G=(\alpha+1) C \varphi_{2} y+G_{0} & H=(\alpha+1) D \varphi_{2} y+H_{0}
\end{array}
$$

where $\varphi_{1}$ is an arbitrary non-constant smooth function on $\mathbb{R}^{2}[w, x], \varphi_{1}(w, x) \neq 0$, and the functions $\varphi_{2}, \varphi_{3}$ are defined by

$$
(\alpha+1)\left(\varphi_{2}\right)^{2}+\left(\varphi_{1}\right)^{2}=\lambda_{2}, \quad-\alpha \varphi_{1} \varphi_{3}=(\alpha+1) \lambda_{3}+\lambda_{2}, \quad d \varphi_{2}(w, x) \neq 0 .
$$

Further, $A_{0}, B_{0}, G_{0}$ and $H_{0}$ are any smooth functions of $w$ and $x$ satisfying the partial differential equations

$$
\begin{aligned}
& \left(A_{0}\right)_{x}^{\prime}-\left(B_{0}\right)_{w}^{\prime}=\left(D A_{0}-C B_{0}\right) \varphi_{2}+\left(D G_{0}-C H_{0}\right)\left(\varphi_{1}-\varphi_{3}\right) \\
& \left(G_{0}\right)_{x}^{\prime}-\left(H_{0}\right)_{w}^{\prime}=\left(D A_{0}-C B_{0}\right)\left(\varphi_{1}+\varphi_{3}\right)-(\alpha+1)\left(D G_{0}-C H_{0}\right) \varphi_{2} .
\end{aligned}
$$

(iii) $(M, g)$ restricted to $V_{q}$ has, in an adapted system of local coordinates, the following form: an orthonormal coframe $\left\{\omega^{1}, \omega^{2}, \omega^{3}\right\}$ is again defined by (1.3) and

$$
\begin{gathered}
A=A(w, x), \quad B=B(w, x), \quad C=\varphi A y+C_{0}, \quad D=\varphi B y+D_{0} \\
G=\frac{1}{2}(\alpha+1) \sqrt{-\lambda_{3}} \varphi A y^{2}+(\alpha+1) \sqrt{-\lambda_{3}} C_{0} y+G_{0} \\
H=\frac{1}{2}(\alpha+1) \sqrt{-\lambda_{3}} \varphi B y^{2}+(\alpha+1) \sqrt{-\lambda_{3}} D_{0} y+H_{0}
\end{gathered}
$$

where $A, B, C_{0}, D_{0}, G_{0}, H_{0}, \varphi$ are arbitrary smooth.functions of $w$ and $x$ satisfying the system of quasilinear partial differential equations

$$
\begin{aligned}
A_{x}^{\prime}-B_{w}^{\prime} & =\sqrt{-\lambda_{3}}\left(A D_{0}-B C_{0}\right) \\
\left(C_{0}\right)_{x}^{\prime}-\left(D_{0}\right)_{w}^{\prime} & =\varphi\left(A H_{0}-B G_{0}\right) \\
\left(G_{0}\right)_{x}^{\prime}-\left(H_{0}\right)_{w}^{\prime} & =-\varphi\left(A D_{0}-B C_{0}\right)+(\alpha+1) \sqrt{-\lambda_{3}}\left(C_{0} H_{0}-D_{0} G_{0}\right) \\
A \varphi_{x}^{\prime}-B \varphi_{w}^{\prime} & =\left(A D_{0}-B C_{0}\right) \alpha \sqrt{-\lambda_{3}} \varphi
\end{aligned}
$$


Here $\varphi=\varphi(w, x)$ is a non-constant function, and the equality $\lambda_{1} \lambda_{3}=\left(\lambda_{2}\right)^{2}$ and the inequalities $\lambda_{1}<0$ and $\lambda_{3}<0$ must be satisfied.

The spaces in the items (i) - (iii) are never locally isometric to each other; in particular, the cases (ii) and (iii) are never locally homogeneous.

Remark 1.2. 1. In the case (iii) of Theorem 1.1 the Ricci eigenvalues satisfy the equality $\rho_{1}^{2}+\rho_{3}^{2}=\rho_{2}\left(\rho_{1}+\rho_{3}\right)$ and the inequalities $\rho_{2}+\rho_{3}<\rho_{1}$ and $\rho_{1}+\rho_{2}<\rho_{3}$.

2. If the condition (G2) is not satisfied, one can find a system of five independent algebraic equations for the six independent connection coefficients $a_{j k}^{i}$. Here $a_{21}^{1}, a_{22}^{1}$ and $a_{31}^{1}$ can be easily eliminated but the remaining two algebraic equations for $a_{23}^{1}, a_{32}^{1}$ and $a_{31}^{2}$ (including the parameters $\alpha$ and $\lambda_{1}, \lambda_{2}, \lambda_{3}$ ) are very complicated and they were printed on six pages by a computer. (We are obliged to B. Fiedler for the computer test.)

3. As it is well-known (see, e.g., [13]), each locally homogeneous Riemannian 3manifold is either locally symmetric or locally isometric to a Lie group with a leftinvariant metric. In the first case, the Ricci eigenvalues cannot be distinct.

4. Obviously, a Riemannian manifold $(M, g)$ with three distinct constant Ricci eigenvalues is locally homogeneous if and only if all connection coefficients $a_{j k}^{i}$ from condition (G1) are constant.

Remark 1.3. A converse to Theorem 1.1/Part(i) also holds in the sense that a 3-dimensional Lie group $G$ with a left-invariant metric $g$ (and with three distinct Ricci eigenvalues) satisfies the conditions (G1) and (G2) for some $\gamma \in\{1,2,3\}$. First, condition $(\mathrm{G} 1)$ is trivially satisfied because all connection coefficients $a_{j k}^{i}$ are constant. As concerns condition (G2), let us mention as obvious fact that a left-invariant vector field $X$ on $(G, g)$ satisfies div $X=0$ if and only if $\mathcal{L}_{X}\left(\omega^{1} \wedge \omega^{2} \wedge \omega^{3}\right)=0$, i.e., if and only if trace $a d(X)=0$ holds on the Lie algebra $\mathcal{G}$. If $G$ is unimodular, then this condition is satisfied for all $X \in \mathcal{G}$. If $G$ is not unimodular, then the Lie algebra $\mathcal{G}$ contains the 2-dimensional unimodular kernel $\mathcal{U}=\{X \in \mathcal{G} \mid$ trace $\operatorname{ad}(X)=0\}$. This kernel is spanned by two vector fields consisting of Ricci eigenvectors, say $E_{2}$ and $E_{3}$ (see [11] for more details). Hence $\operatorname{div} E_{2}=\operatorname{div} E_{3}=0$ on $(G, g)$.

The rest of this paper contains the proof of the previous theorem.

\section{The basic differential equations under the assumptions (G1) and (G2)}

First it is obvious (cf. [7]) that an adapted orthonormal coframe $\left\{\omega^{1}, \omega^{2}, \omega^{3}\right\}$ can be always expressed in the form (1.3) with respect to the adapted local coordinates. Because $\omega^{1} \wedge \omega^{2} \neq 0$ we see that $A D-B C \neq 0$.

From (1.1) we obtain at once, using the standard formulas (see [3])

$$
\left.\begin{array}{l}
d \omega_{2}^{1}+\omega_{3}^{1} \wedge \omega_{2}^{3}=\lambda_{3} \omega^{1} \wedge \omega^{2} \\
d \omega_{3}^{1}+\omega_{2}^{1} \wedge \omega_{3}^{2}=\lambda_{2} \omega^{1} \wedge \dot{\omega}^{3} \\
d \omega_{3}^{2}+\omega_{1}^{2} \wedge \omega_{3}^{1}=\lambda_{1} \omega^{2} \wedge \omega^{3}
\end{array}\right\}
$$$$
\text { where } \omega_{j}^{i}=\sum_{k} a_{j k}^{i} \omega^{k}
$$ 
This leads to a first order partial differential equation.system for the functions $a_{j k}^{i}$ and the coefficients $A, B, C, D, G$ and $H$ from (1.3) (see [7: Chapter 2]).

Now we shall use the conditions (G1) and (G2). From condition (G1) we get at once

$$
a_{j k}^{i}=a_{j k}^{i}(w, x) \quad(1 \leq i, j, k \leq 3) .
$$

Using (1.2) and the definition of the functions $a_{j k}^{i}$ we get $\operatorname{div} E_{3}=\sum_{j} a_{3 j}^{j}=(1+\alpha) a_{31}^{1}$, and hence condition (G2) means

$$
a_{31}^{1}=0 \text { on } \dot{U}_{p}
$$

Therefore our partial differential equation system has a more special form than in [7]. We obtain the nine equations

$$
\begin{aligned}
A_{y}^{\prime} & =C\left(a_{32}^{1}-a_{23}^{1}\right) \\
B_{y}^{\prime} & =D\left(a_{32}^{1}-a_{23}^{1}\right) \\
C_{y}^{\prime} & =A\left(a_{23}^{1}+a_{31}^{2}\right) \\
D_{y}^{\prime} & =B\left(a_{23}^{1}+a_{31}^{2}\right) \\
G_{y}^{\prime} & =(\alpha+1) C a_{21}^{1}-\frac{\alpha+1}{\alpha} A a_{22}^{1} \\
H_{y}^{\prime} & =(\alpha+1) D a_{21}^{1}-\frac{\alpha+1}{\alpha} B a_{22}^{1} \\
G_{x}^{\prime}-H_{w}^{\prime} & =\mathcal{D}\left(a_{32}^{1}-a_{31}^{2}\right)-\frac{\alpha+1}{\alpha} \mathcal{E} a_{22}^{1}+(\alpha+1) \mathcal{F} a_{21}^{1} \\
C_{x}^{\prime}-D_{w}^{\prime} & =\mathcal{D} a_{22}^{1}+\mathcal{E}\left(a_{23}^{1}+a_{31}^{2}\right) \\
A_{x}^{\prime}-B_{w}^{\prime} & =\mathcal{D} a_{21}^{1}+\mathcal{F}\left(a_{32}^{1}-a_{23}^{1}\right)
\end{aligned}
$$

and additional nine equations:

$$
\left.\begin{array}{r}
A\left(a_{21}^{1}\right)_{x}^{\prime}-B\left(a_{21}^{1}\right)_{w}^{\prime}+C\left(a_{22}^{1}\right)_{x}^{\prime}-D\left(a_{22}^{1}\right)_{w}^{\prime}+G\left(a_{23}^{1}\right)_{x}^{\prime} \\
-H\left(a_{23}^{1}\right)_{w}^{\prime}-\mathcal{D}\left(U_{3}-\lambda_{3}\right)-\mathcal{E} V_{3}-\mathcal{F} W_{3}=0 \\
\left(a_{23}^{1}\right)_{w}^{\prime}+A V_{3}+C W_{3}=0 \\
\left(a_{23}^{1}\right)_{x}^{\prime}+B V_{3}+D W_{3}=0
\end{array}\right\}
$$


Here we use the abbreviations

$$
\mathcal{D}=A D-B C, \quad \mathcal{E}=A H-B G, \quad \mathcal{F}=C H-D G
$$

and

$$
\left.\begin{array}{rl}
U_{1} & =\alpha a_{21}^{1} a_{31}^{2}-(\alpha+2) a_{21}^{1} a_{32}^{1} \\
V_{1} & =\frac{(\alpha+1)(\alpha+2)}{\alpha} a_{21}^{1} a_{22}^{1} \\
W_{1} & =\frac{\alpha+1}{\alpha}\left(a_{22}^{1}\right)^{2}-(\alpha+1)^{2}\left(a_{21}^{1}\right)^{2}+a_{23}^{1} a_{31}^{2}-a_{32}^{1} a_{31}^{2}+a_{32}^{1} a_{23}^{1}
\end{array}\right\}
$$

The following formula is a direct consequence of our notation for $\lambda_{i}, \rho_{i}$ and $\alpha$ :

$$
\lambda_{1}+\alpha \lambda_{2}=(\alpha+1) \lambda_{3} .
$$

Due to (1.2) and (2.1) we have only five basic coefficient functions, namely

$$
a_{21}^{1}, \quad a_{22}^{1}, \quad a_{23}^{1}, \quad a_{32}^{1}, \quad a_{31}^{2} .
$$

Substituting the second and the third equations of (2.4) - (2.6) into the first equation (2.4) and using (2.1) we obtain

$$
\left(W_{1}-\lambda_{1}\right)+\alpha\left(V_{2}-\lambda_{2}\right)+(\alpha+1)\left(U_{3}-\lambda_{3}\right)=0 .
$$

By means of the notations (2.7) - (2.9) and formula (2.10), this can be written in the form

$$
a_{23}^{1} a_{31}^{2}-\alpha a_{23}^{1} a_{32}^{1}-(\alpha+1)\left(a_{21}^{1}\right)^{2}-(\alpha+1)\left(a_{22}^{1}\right)^{2}=(\alpha+1) \lambda_{3} .
$$

The differentiation of the second and the third equations of (2.4) - (2.6) with respect to $y$ gives

$$
\begin{array}{lll}
V_{3} A_{y}^{\prime}+W_{3} C_{y}^{\prime}=0 & \left(V_{2}-\lambda_{2}\right) A_{y}^{\prime}+W_{2} C_{y}^{\prime}=0 & V_{1} A_{y}^{\prime}+\left(W_{1}-\lambda_{1}\right) C_{y}^{\prime}=0 \\
V_{3} B_{y}^{\prime}+W_{3} D_{y}^{\prime}=0 & \left(V_{2}-\lambda_{2}\right) B_{y}^{\prime}+W_{2} D_{y}^{\prime}=0 & V_{1} B_{y}^{\prime}+\left(W_{1}-\lambda_{1}\right) D_{y}^{\prime}=0 .
\end{array}
$$

This is a system of linear algebraic equations for $A_{y}^{\prime}, B_{y}^{\prime}, C_{y}^{\prime}$ and $D_{y}^{\prime}$ with the matrix

$$
\mathcal{M}=\left(\begin{array}{ccc}
V_{3} & V_{2}-\lambda_{2} & V_{1} \\
W_{3} & W_{2} & W_{1}-\lambda_{1}
\end{array}\right)
$$

Once given a numeration of the Ricci eigenvalues, we see easily from (2.7) - (2.9) that rank $\mathcal{M}$ is a geometrical invariant. (In fact, the only freedom in the choice of an adapted frame is taking the opposite of any of the vector fields $E_{1}, E_{2}, E_{3}$.) 


\section{Proof of the main result}

In the following we always suppose that $U_{p}$ is a connected and sufficiently small neighbourhood of $p \in M$ such that we can construct our adapted coordinate system $(w, x, y)$ with $E_{3}=\frac{\partial}{\partial y}$ on $U_{p}$. Furthermore, we assume that the restriction of $g$ on $U_{p}$ satisfies the conditions (G1) and (G2) for $\gamma=3$.

Suppose first that $(M, g)$ is locally homogeneous on $U_{p}$. According to the Remark 1.2 Item $3,\left(U_{p}, g\right)$ is locally isometric to a Lie group with a left-invariant metric; this is the case (i) of Theorem 1.1 .

Thus we have to deal only with the cases when the metric is not locally homogeneous. The following Lemma will be very useful in the sequel.

Lemma 3.1. Suppose that $a_{23}^{1}+a_{31}^{2}=0$ and $a_{23}^{1}-a_{32}^{1}=0$ on $U_{p}$. Then all connection coefficients $a_{j k}^{i}$ are constant on $U_{p}$ and the matrix $\mathcal{M}$ must have rank zero on $U_{p}$.

Proof. From (2.11) we obtain on $U_{p}$

$$
\left(a_{22}^{1}\right)^{2}+\left(a_{21}^{1}\right)^{2}+\left(a_{23}^{1}\right)^{2}=-\lambda_{3} .
$$

Differentiate (3.1) with respect to $x$ and $w$. Using (1.2) and (2.12) we get (because of $\mathcal{D}=A D-B C \neq 0$ on $\left.U_{p}\right)$

$$
\begin{gathered}
\frac{\alpha}{\alpha+1} a_{22}^{1}\left(V_{2}-\lambda_{2}\right)-\frac{1}{\alpha+1} a_{21}^{1} V_{1}-a_{23}^{1} V_{3}=0 \\
\frac{\alpha}{\alpha+1} a_{22}^{1} W_{2}-\frac{1}{\alpha+1} a_{21}^{1}\left(W_{1}-\lambda_{1}\right)-a_{23}^{1} W_{3}=0 .
\end{gathered}
$$

Substituting from (2.7) - (2.9) we obtain on $U_{p}$

$$
\begin{aligned}
\frac{\alpha}{\alpha+1} a_{22}^{1} \lambda_{2}=a_{22}^{1} & \left\{-\frac{\alpha+1}{\alpha}\left(a_{22}^{1}\right)^{2}+\frac{\alpha^{2}-\alpha-2}{\alpha}\left(a_{21}^{1}\right)^{2}\right. \\
+ & \left.\left(\frac{\alpha}{\alpha+1}-\frac{2(\alpha+1)}{\alpha}\right)\left(a_{23}^{1}\right)^{2}\right\} \\
-\frac{1}{\alpha+1} a_{21}^{1} \lambda_{1}=a_{21}^{1} & \left\{\left(2 \alpha+1-\frac{1}{\alpha}\right)\left(a_{22}^{1}\right)^{2}+(\alpha+1)\left(a_{21}^{1}\right)^{2}\right. \\
+ & \left.\left(2(\alpha+1)-\frac{1}{\alpha+1}\right)\left(a_{23}^{1}\right)^{2}\right\} .
\end{aligned}
$$

For the further considerations we define the following four sets:

$$
\begin{aligned}
& \mathcal{M}_{1}=\left\{q \in U_{p} \mid a_{22}^{1}(q) a_{21}^{1}(q) \neq 0\right\} \\
& \mathcal{M}_{2}=\left\{q \in U_{p} \mid a_{22}^{1}(q)=0, a_{21}^{\mathrm{i}}(q) \neq 0\right\} \\
& \mathcal{M}_{3}=\left\{q \in U_{p} \mid a_{22}^{1}(q) \neq 0, a_{21}^{1}(q)=0\right\} \\
& \mathcal{M}_{4}=\left\{q \in U_{p} \mid a_{22}^{1}(q)=a_{21}^{1}(q)=0\right\} .
\end{aligned}
$$


We see that $U_{p}=\bigcup_{j=1}^{4} \mathcal{M}_{j}$ and $\mathcal{M}_{i} \cap \mathcal{M}_{j}=\emptyset$ for $i \neq j$.

In the first step we prove that all connection coefficients $a_{j k}^{i}$ are constant on $\mathcal{M}_{j}(j=$ $1, \ldots, 4)$.

Let $q \in \mathcal{M}_{1}$. We can delete the factors $a_{22}^{1}(q), a_{21}^{1}(q)$ in (3.2), (3.3) and (3.1) (3.3) give a system of linear equations for $\left(a_{22}^{1}\right)^{2}(q),\left(a_{21}^{1}\right)^{2}(q),\left(a_{23}^{1}\right)^{2}(q)\left(q \in \mathcal{M}_{1}\right)$. The determinant of this system is equal to $-\frac{(\alpha-1)^{2}}{\alpha}$. If $\alpha=1$, then from (3.2) and (3.3) there follows $\lambda_{1}=\lambda_{2}$ and this is a contradiction. From $\alpha \neq 1$ we find that the solution of our system is independent of $q \in \mathcal{M}_{1}$. Therefore, $\left(a_{22}^{1}\right)^{2},\left(a_{21}^{1}\right)^{2}$ and $\left(a_{23}^{1}\right)^{2}$ are constant on $M_{1}$.

Now, let us suppose $q \in \mathcal{M}_{2}$. Then, in a similar way, (3.1) and (3.3) give a linear equation system for $\left(a_{21}^{1}\right)^{2}(q),\left(a_{23}^{1}\right)^{2}(q) \quad\left(q \in \mathcal{M}_{2}\right)$ with determinant $\frac{\alpha(\alpha+2)}{\alpha+1}$ which is non-zero (indeed, substituting $\alpha=-2$ in this system we get $\lambda_{1}=\lambda_{3}$, i.e. $\rho_{1}=\rho_{3}$ and this is a contradiction). We obtain again that $\left(a_{22}^{1}\right)^{2},\left(a_{21}^{1}\right)^{2}$ and $\left(a_{23}^{1}\right)^{2}$ are constant on $\mathcal{M}_{2}$.

If $q \in \mathcal{M}_{3}$, then (3.1) and (3.2) give a linear equation system for $\left(a_{22}^{1}\right)^{2}(q),\left(a_{23}^{1}\right)^{2}(q)$ with the determinant $-\frac{(2 \alpha+1)}{\alpha(\alpha+1)}$ which is again non-zero. (Substituting $\alpha=-\frac{1}{2}$ in this system we get $\lambda_{2}=\lambda_{3}$, i.e. $\rho_{2}=\rho_{3}$ and this is a contradiction.) This shows that $\left(a_{22}^{1}\right)^{2},\left(a_{21}^{1}\right)^{2}$ and $\left(a_{23}^{1}\right)^{2}$ are constant on $\mathcal{M}_{3}$.

Similarly we find that $\left(a_{22}^{1}\right)^{2},\left(a_{21}^{1}\right)^{2}$ and $\left(a_{23}^{1}\right)^{2}$ are constant on $\mathcal{M}_{4}$.

Because $U_{p}=\bigcup_{j=1}^{4} \mathcal{M}_{j}$ and $a_{j k}^{i}$ are smooth functions on $U_{p}$ we get that $\left(a_{22}^{1}\right)^{2}$, $\left(a_{21}^{1}\right)^{2}$ and $\left(a_{23}^{1}\right)^{2}$ are global constants on $U_{p}$. This and (1.2) show that $a_{33}^{1}, a_{33}^{2}$ and $a_{23}^{1}$ are constant on $U_{p}$. Using the second and the third equation of (2.4)-(2.6) and the inequality $A D-B C \neq 0$ we see that all entries of the matrix $\mathcal{M}$ are zero.

Corollary 3.2. The matrix $\mathcal{M}$ has nowhere rank two.

Proof. Let $p$ be a point of $M$ with rank $\mathcal{M}(p)=2$. Hence there is some neighbourhood $U_{p}$ of $p$ with $\operatorname{rank} \mathcal{M}=2$ on $U_{p}$. Now the system (2.12) implies

$$
A_{y}^{\prime}=B_{y}^{\prime}=C_{y}^{\prime}=D_{y}^{\prime}=0 \text {. on } U_{p} \text {. }
$$

Then, because $A D-B C \neq 0$ on $U_{p}$, the system (2.2) gives

$$
a_{32}^{1}-a_{23}^{1}=a_{23}^{1}+a_{31}^{2}=0 \text { on } U_{p} \text {. }
$$

Now, Lemma 3.1 shows that $\operatorname{rank} \mathcal{M}=0$ on $U_{p}$ and this is a contradiction.

Let $\mathcal{N}_{1}$ be the (open) set of all points of $M$ such that $\operatorname{rank} \mathcal{M}=1$.

Proposition 3.3. There is an open dense subset $\tilde{\mathcal{N}}_{1} \subset \mathcal{N}_{1}$ such that, for each point $p \in \tilde{\mathcal{N}}_{1}$ there exists a neighbourhood $U_{p}$ of $p$ such that the restriction of $g$ to $U_{p}$ is isometric to a generalized Yamato metric (i.e., the case (ii) of Theorem 1.1 occurs).

Proof. Choose $p \in \mathcal{N}_{1}$. Substituting from (2.2) into (2.12), we get easily in a neighbourhood $U_{p}$ of $p$

$$
\left.\begin{array}{rlrl}
\left(a_{23}^{1}+a_{31}^{2}\right) W_{3} & =0 \\
\left(a_{23}^{1}+a_{31}^{2}\right) W_{2} & =0 \\
\left(a_{23}^{1}+a_{31}^{2}\right)\left(W_{1}-\lambda_{1}\right) & =0 & & \left(a_{32}^{1}-a_{23}^{1}\right)\left(V_{2}-\lambda_{2}\right)=0 \\
\left(a_{32}^{1}-a_{23}^{1}\right) V_{1}=0
\end{array}\right\}
$$


and we can also suppose that $\operatorname{rank} \mathcal{M}=1$ on $U_{p}$.

Because, rank $\mathcal{M}>0$, then (2.12) shows that $A_{y}^{\prime} D_{y}^{\prime}-B_{y}^{\prime} C_{y}^{\prime}=0$, and using (2.2) we get

$$
\left(a_{23}^{1}-a_{32}^{1}\right)\left(a_{23}^{1}+a_{31}^{2}\right)=0
$$

on $U_{p}$. Let us suppose that there exist sequences $\left\{q_{m}\right\}_{m \in N}$ and $\left\{\bar{q}_{m}\right\}_{m \in N}$ with $q_{m}, \bar{q}_{m} \in$ $U_{p}(m \in N)$ such that $q_{m}, \bar{q}_{m} \rightarrow p$ and

$$
\left(a_{23}^{1}+a_{31}^{2}\right)\left(q_{m}\right) \neq 0 \text { and }\left(a_{32}^{1}-a_{23}^{1}\right)\left(\bar{q}_{m}\right) \neq 0 \quad \text { for all } m \in N .
$$

Then we get from (3.4)

$$
V_{1}(p)=\left(V_{2}-\lambda_{2}\right)(p)=V_{3}(p)=W_{3}(p)=W_{2}(p)=\left(W_{1}-\lambda_{1}\right)(p)=0
$$

because the functions are continuous. But this is a contradiction to $\operatorname{rank} \mathcal{M}(p)=1$. Therefore we have only the following two possibilities:

a) There is a neighbourhood $\tilde{U}_{p}$ of $p, \tilde{U}_{p} \subset U_{p}$ such that $a_{23}^{1}-a_{32}^{1}=0$ on $\tilde{U}_{p}$.

b) There is a neighbourhood $\tilde{U}_{p}$ of $p, \tilde{U}_{p} \subset U_{p}$ such that $a_{23}^{1}+a_{31}^{2}=0$ on $\tilde{U}_{p}$.

If we interchange the notation in the Case a) (i.e., the basic frame $\left\{E_{1}, E_{2}, E_{3}\right\}$ is replaced by $\left\{\bar{E}_{1}, \bar{E}_{2}, \bar{E}_{3}\right\}$ with $\left.\bar{E}_{1}=E_{2}, \bar{E}_{2}=E_{1}, \bar{E}_{3}=E_{3}\right)$, the new connection coefficients $\bar{a}_{j k}^{i}$ satisfy the equality $\bar{a}_{32}^{2}=0$ (and hence $\bar{a}_{31}^{1}=0$ due to (1.2)) and the conditions $\bar{a}_{23}^{1}+\bar{a}_{31}^{2}=0$ and $\bar{a}_{23}^{1}-\bar{a}_{32}^{1} \neq 0$ on $\tilde{U}_{p}$. Therefore, we can limit ourselves to the Case b).

Thus, let us suppose $a_{23}^{1}+a_{31}^{2}=0$ on $U_{p}$. If $\left(a_{32}^{1}-a_{23}^{1}\right)(q) \neq 0$ for some $q \in U_{p}$, then $V_{1}(q)=\left(V_{2}-\lambda_{2}\right)(q)=V_{3}(q)=0$. On the other hand, if $\left(a_{32}^{1}-a_{23}^{1}\right)(q)=0$, then there is a sequence $q_{m} \rightarrow q, q_{m} \in U_{p}$ with $\left(a_{32}^{1}-a_{23}^{1}\right)\left(q_{m}\right) \neq 0$ for all $m \in N$ (otherwise, due to Lemma 3.1 , we get $\operatorname{rank} \mathcal{M}=0$ in a neighbourhood of $q$, which is a contradiction). This gives $V_{3}\left(q_{m}\right)=\left(V_{2}-\lambda_{2}\right)\left(q_{m}\right)=V_{1}\left(q_{m}\right)$ and from the continuity we get $V_{3}(q)=\left(V_{2}-\lambda_{2}\right)(q)=V_{1}(q)=0$, too.

This shows $V_{1}=V_{2}-\lambda_{2}=V_{3}=0$ on $U_{p}$. From here we get

$$
\begin{aligned}
(\alpha+2) a_{21}^{1} a_{22}^{1} & =0 \\
a_{23}^{1} a_{22}^{1} & =0 \\
(\alpha+1)\left(a_{21}^{1}\right)^{2}-\left(\frac{\alpha+1}{\alpha}\right)^{2}\left(a_{22}^{1}\right)^{2}+\left(a_{23}^{1}\right)^{2} & =\lambda_{2}
\end{aligned}
$$

on $U_{p}$.

Next, let us suppose that there exists a point $q \in U_{p}$ with $a_{23}^{1}(q)=0$. We have two different possibilities:

Case 1: $a_{23}^{1}=0$ on a connected neighbourhood $\bar{U}$ of $q, \bar{U} \subset U_{p}$. Then we get $a_{31}^{2}=0$ on $\bar{U}$. From (3.7) it follows on $\bar{U}$

$$
(\alpha+1)\left(a_{21}^{1}\right)^{2}-\left(\frac{\alpha+1}{\alpha}\right)^{2}\left(a_{22}^{1}\right)^{2}=\lambda_{2}
$$


and from (2.11) we obtain

$$
\left(a_{21}^{1}\right)^{2}+\left(a_{22}^{1}\right)^{2}=-\lambda_{3}
$$

on $\bar{U}$. Solving this equation system, we see that $a_{21}^{1}$ and $a_{22}^{1}$ are constant on $\bar{U}$ and hence all coefficient functions $a_{j k}^{i}$ are constant. Using (2.4) - (2.6) we find that $W_{3}=$ $W_{2}=W_{1}-\lambda_{1}=0$ on $\bar{U}$, but this is a contradiction to rank $\mathcal{M}=1$ on $U_{p}$.

Case 2. There exists a sequence $\left\{q_{m}\right\}_{m \in N}, q_{m} \in U_{p}(m \in N)$ and $q_{m} \rightarrow q$, with $a_{23}^{1}\left(q_{m}\right) \neq 0$ for all $m \in N$. From (3.6) we get $a_{22}^{1}\left(q_{m}\right)=0$ and hence $a_{22}^{1}(q)=0$. Now we know that $a_{23}^{1}(q)=-a_{31}^{2}(q)=a_{22}^{1}(q)=0$. Differentiating (3.7) with respect to $x$ and $w$ at the point $q$ we obtain

$$
\left(a_{21}^{1}\right)(q)\left(a_{21}^{1}\right)_{w}^{\prime}(q)=0 \quad \text { and } \quad\left(a_{21}^{1}\right)(q)\left(a_{21}^{1}\right)_{x}^{\prime}(q)=0
$$

Here $a_{21}^{1}(q) \neq 0$ and hence $\left(a_{21}^{1}\right)_{x}^{\prime}(q)=\left(a_{21}^{1}\right)_{w}^{\prime}(q)=0$ (indeed, if $a_{21}^{1}(q)=0$, then (3.1) and (3.7) imply $\lambda_{3}=\lambda_{2}=0$ and hence $\rho_{2}=\rho_{3}$, which is a contradiction).

Now, from (1.2) we see that $\left(a_{33}^{2}\right)_{x}^{\prime}(q)=\left(a_{33}^{2}\right)_{w}^{\prime}(q)=0$, and from (2.6) it follows $\left(W_{1}-\lambda_{1}\right)(q)=0$. Furthermore, from (2.8) we find $W_{2}(q)=0$. Using (2.5), (1.2) and $W_{2}(q)=0$ we see that $\left(a_{22}^{1}\right)_{x}^{\prime}(q)=\left(a_{22}^{1}\right)_{w}^{\prime}(q)=0$. Now the differentiation of $(2.11)$ at $q$ gives

$$
\left(a_{23}^{1}\right)_{x}^{\prime}(q)\left(a_{32}^{1}\right)(q)=0 \quad \text { and } \quad\left(a_{23}^{1}\right)_{x}^{\prime}(q)\left(a_{32}^{1}\right)(q)=0 .
$$

If $a_{32}^{1}(q)=0$, then $W_{3}(q)=0$ follows from (2.9). On the other hand, if $\left(a_{23}^{1}\right)_{x}^{\prime}(q)=$ $\left(a_{23}^{1}\right)_{w}^{\prime}(q)=0$, we get $W_{3}(q)=0$ again using (2.4). Summarizing, we see that $\operatorname{rank} \mathcal{M}(q)$ $=0$, which is a contradiction.

So, the both :Cases 1) and 2) lead to a contradiction and we must have $a_{23}^{1}(q) \neq 0$ for all $q \in U_{p}$. Then (3.6) gives $a_{22}^{1}=0$ on the whole $U_{p}$.

Summarizing all our considerations we have

$$
a_{22}^{1}=0, \quad a_{23}^{1}+a_{31}^{2}=0, \quad a_{23}^{1} \neq 0 \quad \text { on } U_{p} .
$$

On the other hand (2.8) and (3.8) gives $W_{2}=0$ on $U_{p}$. The formula (3.7) is reduced to

$$
(\alpha+1)\left(a_{21}^{1}\right)^{2}+\left(a_{23}^{1}\right)^{2}=\lambda_{2} .
$$

Using (3.8), (3.9) and (2.11) we obtain

$$
-\alpha a_{23}^{1} a_{32}^{1}=(\alpha+1) \lambda_{3}+\lambda_{2} .
$$

Let us suppose that $a_{23}^{1}=$ const $\neq 0$ on some open subset $V \subset U_{p}$. Then (2.4) shows $W_{3}=0$ on $V$, i.e., more explicitly

$$
a_{21}^{1}\left(\alpha a_{23}^{1}+(\alpha+2) a_{32}^{1}\right)=0
$$

on $V$. Furthermore, $(2.10)$ implies that $W_{1}+(\alpha+1) U_{3}$ is constant on $U_{p}$. In the explicit form,

$$
\left((\alpha+1)^{2}+\alpha+1\right)\left(a_{21}^{1}\right)^{2}+(\alpha+2)\left(a_{23}^{1}\right)^{2}+2 \alpha a_{23}^{1} a_{32}^{1}=\text { const. }
$$


Differentiating (3.11) with respect to $x$ and $w$ we get

$$
\begin{array}{r}
\left(a_{21}^{1}\right)_{x}^{\prime}\left(\alpha a_{23}^{1}+(\alpha+2) a_{32}^{1}\right)+a_{21}^{1}(\alpha+2)\left(a_{32}^{1}\right)_{x}^{\prime}=0 \\
\left(a_{21}^{1}\right)_{w}^{\prime}\left(\alpha a_{23}^{1}+(\alpha+2) a_{32}^{1}\right)+a_{21}^{1}(\alpha+2)\left(a_{32}^{1}\right)_{w}^{\prime}=0
\end{array}
$$

and differentiating (3.12) we obtain

$$
\begin{array}{r}
2\left((\alpha+1)^{2}+\alpha+1\right)\left(a_{21}^{1}\right)_{x}^{\prime} a_{21}^{1}+2 \alpha a_{23}^{1}\left(a_{32}^{1}\right)_{x}^{\prime}=0 \\
2\left((\alpha+1)^{2}+\alpha+1\right)\left(a_{21}^{1}\right)_{w}^{\prime} a_{21}^{1}+2 \alpha a_{23}^{1}\left(a_{32}^{1}\right)_{w}^{\prime}=0
\end{array}
$$

If $\left(\alpha a_{23}^{1}+(\alpha+2) a_{32}^{1}\right)(q) \neq 0$ for some $q \in V$, then $a_{21}^{1}(q)=0$ due to (3.11). Then (3.14) and $a_{23}^{1} \neq 0$ gives $\left(a_{32}^{1}\right)_{x}^{\prime}(q)=\left(a_{32}^{1}\right)_{w}^{\prime}(q)=0$. From $(3.13)$ we see that $\left(a_{21}^{1}\right)_{x}^{\prime}(q)=$ $\left(a_{21}^{1}\right)_{w}^{\prime}(q)=0$. Using (1.2) and (2.6) we get $\left(W_{1}-\lambda_{1}\right)(q)=0$. Summarizing we obtain $\operatorname{rank} \mathcal{M}(q)=0$, which is a contradiction.

Therefore $\alpha a_{23}^{1}+(\alpha+2) a_{32}^{1}=0$ on $V$, i.e. $a_{32}^{1}=$ const on $V$. It follows from (3.12) that $a_{21}^{1}=$ const on $V$. Then all $a_{j k}^{i}$ are constant functions and (2.4) - (2.6) show that rank $\mathcal{M}=0$ on $V$, which is a contradiction.

From these considerations we obtain

$$
a_{23}^{1} \text { is a non-constant function on any open subset } V \subset U_{p} .
$$

Hence and from (3.10) we see that $\alpha a_{23}^{1}+(\alpha+2) a_{32}^{1} \neq 0$ on a dense open subset of $U_{p}$.

If there exists a point $q \in U_{p}$ with $d a_{21}^{1}(q)=0$, i.e. $\left(a_{21}^{1}\right)_{x}^{\prime}(q)=\left(a_{21}^{1}\right)_{w}^{\prime}(q)=$ 0 , then $W_{1}(q)=\lambda_{1}$ from (2.6) and (1.2). But (3.9) shows that $a_{23}^{1}(q)\left(a_{23}^{1}\right)_{x}^{\prime}(q)=$ $a_{23}^{1}(q)\left(a_{23}^{1}\right)_{w}^{\prime}(q)=0$. Because $a_{23}^{1} \neq 0$, we see that $\left(a_{23}^{1}\right)_{x}^{\prime}(q)=\left(a_{23}^{1}\right)_{w}^{\prime}(q)=0$ and this means that $W_{3}(q)=0$. This proves rank $\mathcal{M}(q)=0$ and it is a contradiction to rank $\mathcal{M}=1$ on $U_{p}$. Therefore we have

$$
d a_{21}^{1} \neq 0 \text { on } U_{p} \text { and thus } a_{21}^{1} \neq 0 \text { on a dense open subset of } U_{p} \text {. }
$$

Now, (3.8) - (3.10) and (3.15) are equivalent to (Y1) - (Y4).

Finally, put $\varphi_{1}=a_{23}^{1}, \varphi_{2}=a_{21}^{1}$ and $\varphi_{3}=a_{32}^{1}$. Then from [7] (see, especially, Proposition 9) it follows that the case (ii) of Theorem 1.1 occurs in any neighbourhood $V_{q}$ on which $W_{3} \neq 0$, i.e. $a_{21}^{1}\left(\alpha a_{23}^{1}+(\alpha+2) a_{32}^{1}\right) \neq 0$ holds. The last inequality has a geometrical meaning (once the numeration of $\rho_{i}$ is chosen) and due to (3.15) and (3.16) it holds on a dense open subset of any neighbourhood $U_{p} \subset \mathcal{N}_{1}$. We can define $\widetilde{\mathcal{N}}_{1}=\left\{p \in \mathcal{N}_{1} \mid W_{3}(p) \neq 0\right\}$ and then Proposition 3.3 follows.

Proposition 3.4. Suppose that rank $\mathcal{M}=0$ on a connected open neighbourhood $U_{p} \subset M$ and the restriction of $g$ to $U_{p}$ is not locally homogeneous. Then the restriction of $g$ to $U_{p}$ is isometric to a metric for which $a_{22}^{1}=a_{23}^{1}=a_{32}^{1}=0,\left(a_{21}^{1}\right)^{2}=-\lambda_{3}$ on $U_{p}$ and $a_{31}^{2}$ is an arbitrary non-constant smooth function.

Proof. The assumptions give

$$
V_{3}=0, \quad V_{2}=\lambda_{2}, \quad V_{1}=0, \quad W_{3}=0, \quad W_{2}=0, \quad W_{1}=\lambda_{1}
$$


on $U_{p}$. From $(2.10)$ we get on $U_{p}$

$$
U_{3}=\lambda_{3} \text {. }
$$

Using the second and the third equation of (2.4) - (2.6) and also (1.2) we obtain on $U_{p}$ that

$$
a_{23}^{1}=\text { const } ; \quad a_{33}^{1}=\text { const }, \quad a_{22}^{1}=\text { const }, \quad a_{33}^{2}=\text { const }, \quad a_{21}^{1}=\text { const } .
$$

The equations $V_{1}=0$ and $W_{2}=0$ give

$$
(\alpha+2) a_{21}^{1} a_{22}^{1}=0 \quad \text { and } \quad(2 \alpha+1) a_{21}^{1} a_{22}^{1}=0
$$

on $U_{p}$. This shows that

$$
a_{21}^{1} a_{22}^{1}=.0
$$

on $U_{p}$. We have the following three possible cases for the constants $a_{22}^{1}$ and $a_{21}^{1}$ :
(a) $a_{22}^{1}=a_{21}^{1}=0$
(b) $a_{22}^{1} \neq 0, a_{21}^{1}=0$
(c) $a_{22}^{1}=0, a_{21}^{1} \neq 0$.

Case (a): Here (3.17) and (3.18) give

$$
\begin{aligned}
a_{32}^{1} a_{23}^{1}-a_{32}^{1} a_{31}^{2}+a_{23}^{1} a_{31}^{2} & =\lambda_{1} \\
-a_{32}^{1} a_{23}^{1}-a_{32}^{1} a_{31}^{2}-a_{23}^{1} a_{31}^{2} & =\lambda_{2} \\
-a_{32}^{1} a_{23}^{1}+a_{32}^{1} a_{31}^{2}+a_{23}^{1} a_{31}^{2} & =\lambda_{3} .
\end{aligned}
$$

It follows

$$
a_{32}^{1} a_{31}^{2}=-\frac{1}{2}\left(\lambda_{1}+\lambda_{2}\right), \quad a_{23}^{1} a_{31}^{2}=\frac{1}{2}\left(\lambda_{1}+\lambda_{3}\right), \quad a_{23}^{1} a_{32}^{1}=-\frac{1}{2}\left(\lambda_{2}+\lambda_{3}\right) .
$$

If $\lambda_{1}+\lambda_{3} \neq 0$ or $\lambda_{2}+\lambda_{3} \neq 0$, then $a_{23}^{1} \neq 0$. Because $a_{23}^{1}=$ const, we get that $a_{32}^{1}$ and $a_{31}^{2}$ are constant on $U_{p}$. But in this case, all connection coefficients are constant on $U_{p}$ and the metric would be locally homogeneous. On the other hand, $\lambda_{1}+\lambda_{3}=\lambda_{2}+\lambda_{3}=0$ is not possible. This shows that the case (a) does not occur.

Case (b): If we interchange the notation (i.e., the basic frame $\left\{E_{1}, E_{2}, E_{3}\right\}$ is replaced by $\left\{\bar{E}_{1}, \bar{E}_{2}, \bar{E}_{3}\right\}$ with $\bar{E}_{1}=E_{2}, \bar{E}_{2}=E_{1}$ and $\left.\bar{E}_{3}=E_{3}\right)$, the new connection coefficients $\bar{a}_{j k}^{i}$ satisfy $\bar{a}_{32}^{2}=0, \bar{a}_{31}^{1}=0, \bar{a}_{22}^{1}=0, \bar{a}_{21}^{1} \neq 0, \bar{a}_{23}^{1}=$ const on $U_{p}$. Therefore, it is sufficient to handle the case (c).

Case (c): From (3.17) and (3.18) we obtain

$$
\begin{aligned}
-(\alpha+1)^{2}\left(a_{21}^{1}\right)^{2}+a_{31}^{2} a_{23}^{1}-a_{32}^{1} a_{31}^{2}+a_{32}^{1} a_{23}^{1} & =\lambda_{1} \\
(\alpha+1)\left(a_{21}^{1}\right)^{2}-a_{32}^{1} a_{23}^{1}-a_{32}^{1} a_{31}^{2}-a_{23}^{1} a_{31}^{2} & =\lambda_{2} \\
-\left(a_{21}^{1}\right)^{2}+a_{31}^{2} a_{23}^{1}-a_{23}^{1} a_{32}^{1}+a_{32}^{1} a_{31}^{2} & =\lambda_{3} \\
-\alpha a_{23}^{1}-(\alpha+2) a_{32}^{1} & =0 .
\end{aligned}
$$

If $\alpha=-2$, we get $a_{23}^{1}=0$ and this shows that $\lambda_{1}=\lambda_{2}$, which is impossible. Therefore we can suppose $\alpha \neq-2$. Then (3.22) shows that $a_{32}^{1}=$ const: on $U_{p}$. The addition of (3.19) and (3.21) gives

$$
-\left(1+(\alpha+1)^{2}\right)\left(a_{21}^{1}\right)^{2}+2 a_{23}^{1} a_{31}^{2}=\lambda_{1}+\lambda_{3},
$$

i.e. if $a_{23}^{1} \neq 0$, then $a_{31}^{2}:=$ const on $U_{p}$ and the restriction of $g$ onto $U_{p}$ is locally homogeneous. Therefore $a_{23}^{1}=0$ on $U_{p}$. Now (3.22) gives $a_{32}^{1}=0$ on $U_{p}$ since $\alpha \neq-2$. Then, (3.21) shows $\left(a_{21}^{1}\right)^{2}=-\lambda_{3}$. 
For the eigenvalues in the last case we get $\lambda_{2}=-(\alpha+1) \lambda_{3}$ and $\lambda_{1}=(\alpha+1)^{2} \lambda_{3}$, and this is equivalent to the single equation $\lambda_{1} \lambda_{3}=\lambda_{2}^{2}$.

Proposition 3.5. Under the assumptions of Proposition 3.4, the metric $g$ restricted on $U_{p}$ is given by the case (iii) of our Theorem.

Proof. From $\lambda_{1} \lambda_{3}=\lambda_{2}^{2}$ and $\left(a_{21}^{1}\right)^{2}=-\lambda_{3}$ we get $a_{21}^{1} \neq 0$ on $U_{p}$. Furthermore, we have $U_{1}=\alpha a_{21}^{1} a_{31}^{2}$. The equations (2.4) - (2.6) are automatically satisfied except the first equation of (2.6) which gives

$$
A\left(a_{31}^{2}\right)_{x}^{\prime}-B\left(a_{31}^{2}\right)_{w}^{\prime}=\mathcal{D} \alpha a_{21}^{1} a_{31}^{2} .
$$

Now, the integration of the equations (2.2) gives

$$
\left.\begin{array}{rl}
A_{y}^{\prime} & =B_{y}^{\prime}=0 \text { on } U_{p}, \text { i.e. } A=A(w, x), B=B(w, x) \\
C & =A a_{31}^{2} y+C_{0}, \quad D=B a_{31}^{2} y+D_{0} \\
G & =\frac{1}{2}(\alpha+1) a_{21}^{1} a_{31}^{2} A y^{2}+(\alpha+1) a_{21}^{1} C_{0} y+G_{0} \\
H & =\frac{1}{2}(\alpha+1) a_{21}^{1} a_{31}^{2} B y^{2}+(\alpha+1) a_{21}^{1} D_{0} y+H_{0}
\end{array}\right\}
$$

where $C_{0}, D_{0}, G_{0}$ and $H_{0}$ are functions of $w$ and $x$ only. From (3.23) we conclude

$$
\begin{aligned}
& \mathcal{D}=A D_{0}-B C_{0} \\
& \mathcal{E}=\left(A D_{0}-B C_{0}\right)(\alpha+1) a_{21}^{1} y+A H_{0}-B G_{0} \\
& \mathcal{F}=\frac{1}{2}(\alpha+1) a_{21}^{1} a_{31}^{2}\left(A D_{0}-B C_{0}\right) y^{2}+\left(A H_{0}-B G_{0}\right) a_{31}^{2} y+C_{0} H_{0}-D_{0} G_{0} .
\end{aligned}
$$

Now, the equations (2.3) and the first equation of (2.6) can be written as a system of partial differential equations:

$$
\begin{aligned}
A_{x}^{\prime}-B_{w}^{\prime} & =a_{21}^{1}\left(A D_{0}-B C_{0}\right) \\
\left(C_{0}\right)_{x}^{\prime}-\left(D_{0}\right)_{w}^{\prime} & =a_{31}^{2}\left(A H_{0}-B G_{0}\right) \\
\left(G_{0}\right)_{x}^{\prime}-\left(H_{0}\right)_{w}^{\prime} & =-a_{31}^{2}\left(A D_{0}-B C_{0}\right)+(\alpha+1) a_{21}^{1}\left(C_{0} H_{0}-D_{0} G_{0}\right) \\
A\left(a_{31}^{2}\right)_{x}^{\prime}-B\left(a_{31}^{2}\right)_{w}^{\prime} & =\left(A D_{0}-B C_{0}\right) \alpha a_{21}^{1} a_{31}^{2} .
\end{aligned}
$$

Substituting $a_{21}^{1}=\sqrt{-\lambda_{3}}$ and $a_{31}^{2}=\varphi$ we get the case (iii) of Theorem 1.1.

Remark 3.6. (a) In the system (3.24) the functions $B, D_{0}$ and $H_{0}$ can be chosen as arbitrary functions; the remaining equations can be solved by using the CauchyKowalewski theorem. Each particular solution depends on four arbitrary functions of one variable.

(b) An explicit solution of system (3.24) is given by

$$
\begin{aligned}
B & =H_{0}=0, \quad D_{0}=1, \quad C_{0}=0 \\
A & =\exp \left(\sqrt{-\lambda_{3}} x\right), \quad G_{0}=-\frac{1}{2(\alpha+1) \sqrt{-\lambda_{3}}} \exp \left((\alpha+1) \sqrt{-\lambda_{3}} x\right) \\
a_{31}^{2} & =\exp \left(\alpha \sqrt{-\lambda_{3}} x\right) .
\end{aligned}
$$


(c) The condition $\lambda_{1} \lambda_{3}=\lambda_{2}^{2}$ gives for the Ricci eigenvalues the relations

$$
\rho_{1}^{2}+\rho_{3}^{2}=\rho_{2}\left(\rho_{1}+\rho_{3}\right), \quad \rho_{2}+\rho_{3}<\rho_{1}, \quad \rho_{1}+\rho_{2}<\rho_{3} .
$$

Proposition 3.7. The rank zero examples given by the case (iii) of Theorem 1.1 are never locally isometric to the generalized Yamato examples given by the case (ii) of Theorem 1.1.

Proof. Assume a local isometry between a generalized Yamato example and an example given by (3.23). In the second case we shall denote the corresponding geometrical quantities by bars. The Ricci eigenvalues are the same. Now, via the local isometry we must have $\bar{\omega}^{i}=\epsilon_{i} \omega^{i} \quad\left(i=1,2,3 ; \epsilon_{i}= \pm 1\right)$. Especially, we get

$$
\bar{x}=\bar{x}(w, x), \quad \bar{w}=\bar{w}(w, x), \quad \bar{y}=\epsilon_{3} y+\Phi(w, x)
$$

as a local expression for our isometry. The equation $\bar{w}^{3}=\epsilon_{3} \omega^{3}$ implies $\bar{G} d \bar{w}+\bar{H} d \bar{x}=$ $\epsilon_{3}(G d w+H d x)-d \Phi$. Now, substitute for $G, H, \bar{G}$ and $\bar{H}$ the corresponding expressions from (ii) and (iii) of Theorem 1.1 and also substitute for $\bar{y}$ from (3.25). According to (3.25), the differentials $d \bar{x}$ and $d \bar{w}$ are linear combinations of $d x$ and $d w$ with coefficients not depending on $y$. Then we can compare the 1-forms which are coefficients of $y^{2}$. We obtain $\bar{A} d \bar{w}+\bar{B} d \bar{x}=0$, i.e. $\bar{\omega}^{1}=0$, which is a contradiction .

Finally, we shall complete the proof of the main Theorem 1.1. We put

$$
\begin{aligned}
& \mathcal{N}_{1}=\{p \in M \mid \operatorname{rank} \mathcal{M}(p)=1\} \\
& \tilde{\mathcal{N}}_{1}=\left\{p \in \mathcal{N}_{1} \mid W_{3}(p) \neq 0\right\} \\
& \mathcal{N}_{2}=\left\{p \in M \mid \operatorname{rank} \mathcal{M}=0 \text { on some open connected neighbourhood } U_{p}\right\}
\end{aligned}
$$

Then $\mathcal{N}=\tilde{\mathcal{N}}_{1} \cup \mathcal{N}_{2}$ is an open dense subset of $M$ and Theorem 1.1 follows from the previous propositions and arguments.

Acknowledgement. The authors are obliged to the referees and to the editors for the careful inspection of the original paper and for improving its style.

\section{References}

[1] Bianchi, L.: Sugli spazi normali a tre dimensioni colle curvature principali costanti. In: Opere, vol. IX, 214-224, Cremonese, Roma 1958.

[2] DeTurck, D. M.: Existence of metrics with prescribed Ricci curvature: Local theory. Invent. Math. 65 (1981), 179 - 207.

[3] Kobayashi, S. and K. Nomizu: Foundations of Differential Geometry. Vol. I. New York: Intersci. Publ. 1963.

[4] Kowalski, O.: An explicit classification of 3-dimensional Riemannian spaces satisfying $R(X, Y) \cdot R=0$. Preprint 1991 . 
[5] Kowalski, O.: A classification of Riemannian 3-manifolds with constant principal Ricci curvatures $\rho_{1}=\rho_{2} \neq \rho_{3}$. Nagoya Math. J. 132 (1993), 1 - 39.

[6] Kowalski, O.: Nonhomogeneous Riemannian 3-manifolds with distinct constant Ricci eigenvalues. Comment. Math. Univ. Carolinae 34 (1993), 451 - 457.

[7] Kowalski, O. and F. Prüfer: On Riemannian 3-manifolds with distinct constant Ricci eigenvalues. Math. Ann. (1994), $17-28$.

[8] Kowalski, O., Tricerri F. and L. Vanhecke: New examples of non-homogeneous Riemannian manifolds whose curvature tensor is that of a Riemannian symmetric space. C. R. Acad. Sci. Paris (Sér. l) 311 (1990), 355 - 360.

[9] Kowalski, O., Tricerri, F. and L. Vanhecke: Curvature homogeneous Riemannian manifolds. J. Math. Pures Appl. 71 (1992), 471 - 501.

[10] Kowalski, O., Tricerri F. and L. Vanhecke: Curvature homogeneous spaces with a solvable Lie group as a homogeneous model. J. Math. Soc. Japan 44 (1992), 461 - 484.

[11] Milnor, J.: Curvatures of left invariant metrics on Lie groups. Adv. Math. 21 (1976), $293-329$.

[12] Sekigawa, K.: On some 3-dimensional Riemannian manifolds. Hokkaido Math. J. 2 (1973), $259-270$.

[13] Sekigawa; K.: On some 3-dimensional curvature homogeneous spaces. Tensor (N.S.):31 (1977), 87 - 97.

[14] Singer, I. M.: Infinitesimally homogeneous spaces. Comm. Pure Appl. Math. 13 (1960), $685-697$.

[15] Spiro, A. and F. Tricerri: 3-dimensional Riemannian metrics with prescribed Ricci principal curvatures. J. Math. Pures Appl. (to appear).

[16] Tsukada, T.: Curvature homogeneous hypersurfaces immersed in a real space form. Tôhoku Math. J. 40 (1988), 221 - 244.

[17] Yamato, K.: A characterization of locally homogeneous Riemannian manifolds of dimension three. Nagoya Math. J. 123 (1991), 77 - 90.

Received 24.05.1994 